This item was submitted to Loughborough's Research Repository by the author.

Items in Figshare are protected by copyright, with all rights reserved, unless otherwise indicated.

\title{
Delayed peroneal muscle reaction time in male amateur footballers during a simulated prolonged football protocol
}

\section{PLEASE CITE THE PUBLISHED VERSION}

https://doi.org/10.1080/15438627.2020.1868467

\section{PUBLISHER}

Taylor \& Francis (Routledge)

\section{VERSION}

AM (Accepted Manuscript)

\section{PUBLISHER STATEMENT}

This is an Accepted Manuscript of an article published by Taylor \& Francis in Research in Sports Medicine on 29 Dec 2020, available online: https://doi.org/10.1080/15438627.2020.1868467

\section{LICENCE}

CC BY-NC-ND 4.0

\section{REPOSITORY RECORD}

Sun, Wei, Edwin Chan, and Daniel Fong. 2020. "Delayed Peroneal Muscle Reaction Time in Male Amateur Footballers During a Simulated Prolonged Football Protocol”. Loughborough University. https://hdl.handle.net/2134/13416815.v1. 


\title{
Delayed peroneal muscle reaction time in male amateur footballers during simulated prolonged football protocol
}

Wei Sun ${ }^{1}$, Edwin C.H. Chan ${ }^{2}$, Daniel T.P. Fong ${ }^{2, *}$

1. College of Sports and Health, Shandong Sport University, Jinan, Shandong, China

2. National Centre for Sport and Exercise Medicine, School of Sport, Exercise and Health Sciences, Loughborough University, Loughborough, UK

* Corresponding author: Dr Daniel T.P. Fong (d.t.fong@lboro.ac.uk)

\begin{abstract}
Peroneal muscle fatigue could result in ankle inversion sprain injuries. This study investigated the peroneal muscle reaction time during a simulated prolonged football protocol. Nine male footballers completed a 105-minute simulated prolonged football protocol. The peroneal muscle reaction time to an ankle inversion perturbation was measured every 15 minutes by a surface electromyography system sampling at $1000 \mathrm{~Hz}$. One-way repeated ANOVA with post-hoc paired t-test showed a steady upward trend starting from $48.9 \mathrm{~ms}$ at baseline to $57.1 \mathrm{~ms}$ at the end of the first half, followed by a recovery back to $50.9 \mathrm{~ms}$ at the start of the second half and a further delay in the last 30 minutes to $60.2 \mathrm{~ms}$ at the end of the protocol. Delayed peroneal muscle reaction was found after 30 minutes of the first half and 15 minutes of the second half of a football match. The risk of ankle sprain could increase in the latter minutes in each half protocol. Thus, prevention injury training strategies should focus on these specific durations in football matches.
\end{abstract}

Key words: ankle sprain, syndesmotic injuries, ligamentous injuries, neuromuscular control, Soccer 


\section{Introduction}

Ankle sprain was the most common injury in football, accounting for approximately $84 \%$ of all sports-related injuries (Fong et al, 2007; Doherty et al, 2014). Football players suffering from repeated ankle sprains are more likely to develop chronic ankle instability (Gribble et al, 2016). The recurrence rate for a lateral ankle sprain is reportedly as high as $80 \%$ among athletes, and it is responsible for the longest absenteeism from participation compared with that in other sports injuries (Fong et al, 2007). Ankle injuries also have a drastic effect on the healthcare system, with an estimation of 1-1.5 million people in the UK attending emergency rooms and clinics yearly (Lamb et al, 2009) and medical costs amounting to £1-2 billion annually. In the US and the Netherlands, such costs were estimated to be US\$ 6.2 billion and $€ 208$ million per year, respectively (Gribble et al, 2016).

A previous research pointed out that one common cause of ankle sprain injury is delayed peroneal muscle reaction time to an ankle inversion perturbation (Fong et al, 2009). Peroneal muscle reaction time was defined as the time between ankle perturbation and the onset of peroneal muscle activity; it could be obtained by measuring the timing of peroneal activation in response to a sudden inversion perturbation by using electromyography and a trapdoor mechanism (Hoch \& McKeon, 2014). When the ankle joint is forced into excessive inversion, the peroneal muscle is the first to react by providing a reflexive contraction as a dynamic defense mechanism to protect the ankle joint (Hertel, 2002). Some studies showed that people with ankle sprain exhibited delayed peroneal reaction time to inversion perturbation compared with healthy ones (Hoch \& McKeon, 2014). The peroneal muscle reaction time was found to be around 55-80 ms (Konradsen and Ravn, 1991), which is still too slow to react to a sudden inversion ankle sprain that occurred in 40-50 ms (Fong et al, 2009). Further understanding of the peroneal muscle reaction time during football games could help introduce ankle training strategies for 
preventing injuries.

Previous studies also suggested that ankle sprains are more likely to occur during the latter minutes of the first half and during the second half in a football game (de Noronha et al, 2019). Football players were reported to experience muscular fatigue after a 90-minute football match as they were required to perform a lot of running, changing direction, jumping, and kicking that would lead to muscular fatigue (Reilly et al, 2008). Fatigue from prolonged matches may affect the muscle responses, motor control and joint position sense for maintaining joint stability (Gribble et al, 2007), induce a change in balance strategy and increased the ankle muscles reaction time and as a result (Valldecabred et al, 2020), increase the risk of ankle sprain and decrease the level of performance (Almonroeder et al, 2020). Those findings may be able to explain the reason why more ankle sprain injuries were being recorded towards the end of each half in real football matches (de Noronha et al, 2019). However, the delayed reaction time with prolonged football game is still unclear, it is very essential to explore the correlation between delayed reaction time and prolonged football game for designing the appropriate training methods for preventing ankle sprain.

Some studies have tried to use isokinetic exercise (Jackson et al, 2009) and football-specific intermittent exercise (Rahnama et al, 2006) protocols to introduce fatigue to the ankle muscles. However, these protocols do not truly represent the nature of football, which is a prolonged exercise with a mixture of different exercise intensities, i.e., running, jogging, and sprinting. Therefore, the present study aimed to investigate the peroneal muscle reaction time in male amateur football players during a simulated prolonged football protocol. The peroneal muscle reaction time to an ankle inversion perturbation was hypothesized to significantly increase from baseline in a simulated prolonged football protocol. 


\section{Materials and methods}

\section{Participant}

Nine healthy male football players (age: $23.3 \pm 0.8$ years; height: $1.77 \pm 0.04 \mathrm{~m}$; body mass: $72.5 \pm 8.4 \mathrm{~kg}$ ) from a local amateur football league were recruited using invitation letters, advertisements and personal invitations in this study. All participants had at least 5 years of recreational football experience, and were members of several football teams in a local amateur league with teams from local universities and colleges. The exclusion criteria included balance disorders, serious lower extremity injury within 1 year, ankle instability, and sensory impairments, as evaluated by an orthopedic specialist. All the participants did not perform any vigorous exercise 24 hours before testing. All the participants did not perform any vigorous exercise 24 hours before testing. They completed a health screen questionnaire and a written informed consent form approved by the Loughborough University Research Ethics Committee (R18-P069).

\section{Sample size calculation}

Sample size estimation was performed on G*Power software (Germany) on the basis of a previous study, which reported that the peroneal reaction time increased from $40.76 \pm 10.90 \mathrm{~ms}$ to $53.74 \pm 4.76 \mathrm{~ms}$ after a football game (Fong et al, 2020). By setting the level of significance to 0.05 and the statistical power to 0.80 in a two-tailed test on matched pairs, the effect size and estimated required sample size were calculated to be 1.37 and 7 , respectively.

\section{Ankle peroneal muscle reaction test}

This test required the participant to stand with their dominant leg on a trapdoor platform which has been widely used in studies to produce a simulate an ankle sprain motion and the other leg on 
a fixed block with their body weight placed equally on their feet. The dominant limb was defined as the preferred limb to kick a ball as verbally reported by the participant (Sun et al, 2015). The sudden simulated ankle inversion perturbation was introduced to the dominant limb for EMG data collection for three trials. The sEMG data collections of the peroneal muscle were conducted at $1000 \mathrm{~Hz}$, using the Trigno Wireless System (Delsys Inc., Boston, MA, USA). The use of a $1000 \mathrm{~Hz}$ sampling rate was suggested to have a moderate to high test-retest reliability for testing muscle latency, with interclass correlation coefficients ranging from 0.68-0.94 (Xu et al, 2005). The amplifier bandwidth frequency ranged from 20 to $450 \mathrm{~Hz}$, with an input voltage of 9 VDC at $0.7 \mathrm{~A}$ and the common-mode rejection ratio was $80 \mathrm{Db}$. A wireless Trigno EMG sensor was attached on the skin surface of the peroneal muscle belly (upper 1/4 position between the tip of the head of the fibula to the tip of the lateral malleolus) on the lateral side of the lower limb by a method in an EMG manual (Figure 1) (Perotto, 2005; Fong et al, 2013). Recording sites were prepared by shaving the area and wiping with alcohol pads to decrease electrical impedance, Electrodes $(41 \times 20 \times 5 \mathrm{~mm}, \quad$ D.E. 2.3, Delsys Inc., Boston, MA) were placed along the longitudinal axis of peroneal muscle on the dominant leg of the participant's body (Gullet et al, 2009).

EMG data were analyzed in accordance with the procedures from the International Society of Electrophysiology and Kinesiology (Merletti.R.,1999) by using the EMG Works Analysis 4.0 (Delsys Inc., Boston, MA, USA). To calculate the mean normalized EMG values, the raw EMG signals were subsetted, filtered (passband: 3, response: band pass, corner F1: $10 \mathrm{~Hz}$, corner F2: $500 \mathrm{~Hz}$ ), rectified, integrated (root mean square (window length: 0.100, window overlap: 0.08, remove offset) to calculate the mean normalized EMG values. The onset time of the peroneal muscle was determined by a sudden increase of EMG signal which exceeded $5 \%$ of the maximum signal value of the muscle (Konrad, 2006; Fong, et al., 2020). The time between the 
start of pulling the trigger of the trapdoor platform and the onset of the EMG signal was the reaction time of the peroneal muscle. The average value of the reaction time from three trials was used for statistical analysis.

\section{Prolonged simulated football protocol}

The prolonged simulated football protocol was modified from the Loughborough intermittent shuttle test to simulate a football game (Nicholas et al, 2000). The shuttle running test includes two 45 minutes half with a 15 minutes rest period in between. The test was set up with two cones $20 \mathrm{~m}$ apart and each cycle of the test includes $60 \mathrm{~m}$ walking. $20 \mathrm{~m}$ sprinting, 4 seconds recovery walk, and $60 \mathrm{~m}$ jogging and $60 \mathrm{~m}$ sprinting (Fong et al, 2020). Each participant started with a dynamic warmup for 15 minutes and then moved on to the ankle muscle reaction test for the EMG data collection at 0 minutes before starting the prolonged simulated football protocol. The peroneal reaction test was then repeated at every 15 minutes interval, i.e. at $0,15,30,45,60,75$, 90, and 105 minutes for EMG data collection. The participants were monitored by the researcher throughout the shuttle running test and required to repeat the cycle until each 15 minutes interval was reached. No practice trials were allowed to ensure the unexpected nature of an ankle sprain.

\section{Statistical analysis}

Statistical analysis was performed with SPSS software (version 20.0, SPSS Inc, USA) in this study. All the data were reported as means \pm standard deviations. One-way repeated ANOVA was conducted on the dependent variables over time. If a significant time effect was found, a post-hoc Bonferroni test was conducted to evaluate the significant difference between reaction time at each time and baseline. The significance level was set at $\mathrm{p}<0.05$. Partial eta squared $\left(\mathrm{n}^{2} \mathrm{p}\right)$ was used to represent the effect of the time effect of repeated ANOVA. The thresholds for partial eta squared were as follows: 0.01-0.06, small; 0.06-0.14, moderate; >0.14, large (Pierce et al, 
2004). Cohen's d was used to represent the effect size of post hoc comparison. The thresholds for Cohen's d were as follows: $<0.2$, trivial; 0.21-0.50, small; 0.510.80, medium; >0.81, large (Cohen, 2013).

\section{Results}

As shown in Table 1 and Figure 2, one-way repeated ANOVA suggested the significant time effect for the reaction time $\left(\mathrm{F}=38.937, \mathrm{p}<0.001, \mathrm{n}^{2} \mathrm{p}=0.848\right)$. The post-hoc results showed that the reaction time at $30(p=0.001$, Cohen's $d=4.05), 45(p<0.001$, Cohen's $d=5.37), 75(p=$ 0.005, Cohen's $d=3.37), 90(p<0.001$, Cohen's $d=6.91)$, and $105 \min (p<0.001$, Cohen's $d=$ 9.13) throughout the prolonged simulated football protocol increased significantly compared with that at baseline.

\section{Discussion}

The results showed that the peroneal muscle reaction time significantly increased at $30,45,60$, and 90 minutes from the beginning, thereby supporting the hypothesis. The results were similar and supportive to those of most previous studies that tested peroneal muscle reaction time in a sudden simulated ankle inversion sprain situation (Henry et al, 2010). These findings suggested that muscular or physiological fatigue may have been induced at the later stage of each half of the prolonged exercise protocol and caused an increase in peroneal muscle reaction time. Although the range of the peroneal muscle reaction time in the present study stayed in the healthy range at 55-80 ms (Konradsen \& Ravn, 1991), it was still not quick enough to react to a sudden ankle inversion that occurred within $50 \mathrm{~ms}$ (Fong et al, 2009). Moreover, the injury was expected to occur faster in dynamic motions, such as in football games, and with a higher twisting force; thus, an even faster peroneal reaction time was needed for protection. The increase in peroneal muscle reaction time towards the end of each half could also increase the 
ankle injury risk at the later stage of each half.

A notable detail that the reaction time significantly increased at $75(56.6 \pm 3.0 \mathrm{~ms}), 90(59.2 \pm$ $1.6 \mathrm{~ms})$, and $105(60.2 \pm 1.1 \mathrm{~ms})$ minutes in the second half in this study, while the previous study (Fong et al, 2020) found the reaction time significantly increased only at 105 minutes, the end of a football game. The gender might lead to a discrepancy in the increasing reaction time of the second half. The male participants were recruited in this study, female in a previous study. A recent study showed that the maximal isometric peak force recovered in the male group was significantly slower during the first hour of rest than that in the female group after prolonged exercise-induced fatigue (Hakkinen, 1993). This finding may imply that the potential risk of ankle sprain was larger in males than in females during the second half of the football game. Another interesting finding was that the peroneal reaction time (from $48.9 \mathrm{~ms}$ to $60.2 \mathrm{~ms}$ ) of each time point in the present study was longer than that in the previous study $(40.76-53.74 \mathrm{~ms})$. The different methods to define the starting time of the reaction time may lead to the discrepancy between the two studies. Previous studies found that two definitions were adopted: first was between the start of pulling the trigger of the trapdoor platform and the first rising response of EMG signals (Sun et al, 2016) and the second one was between the start of ankle inversion motion and the first rising response of EMG signals (Fong et al, 2020).

In addition, the peroneal muscle reaction time on the dominant leg was examined in the present study. The study that investigated the delayed reaction time during prolonged football protocol in female amateur footballers (Fong et al, 2020) showed a significantly shorter peroneal latency in the nondominant leg than in the dominant leg. The difference may be the result of different demands being placed on the dominant and nondominant legs during exercise (Beynnon et al, 2002). For example, football players shoot, pass and jump with their dominant leg more than 
their nondominant leg; therefore, the dominant leg may experience fatigue faster and result in a longer peroneal latency (Knight \& Weimar, 2011). This phenomenon could explain why the ankle sprain incident was 2.4 times more on the dominant leg than on the non-dominant leg in a competitive football season (Yeung et al, 1994). However, the reaction time of the nondominant leg during prolonged football protocol is still unclear. Further study could focus on the difference in the reaction time between these legs during prolonged football exercise protocol for differentiated training strategy of ankle sprain injury prevention. Peroneal muscle endurance should be evaluated in the preseason screening test (Delvaux et al, 2020). Some training interventions, such as the recent successful attempts of the use of kinesiology tape (Farquharson \& Greig, 2017) and compression stockings (Pavin et al, 2019), could also be conducted to reduce the peroneal muscle reaction time and prevent ankle sprain injuries.

This study has three limitations. First, the trapdoor platform for an ankle sprain simulation has caused some concern about its validity as most of the ankle sprain injuries in football normally happened in dynamic motions such as landing from a jump and running instead of standing with both feet flat on a flat surface. However, this is the most common way among the very few methods to mimick an ankle sprain motion for the peroneal muscle reaction test. Second, there was potential human response error on the synchronisation of EMG data collection for calculation of the peroneal muscle reaction time as there may be a minor time error between pulling the trigger of the trapdoor platform and the real starting time of ankle inversion motion. This finding implies that the standard methods of data collection and definition of reaction time are very important to assess the neuromuscular reaction function in the future study. Therefore, the researcher should focus on standardizing the testing methods on neuromuscular reaction time for conclusions from many studies, such as sampling frequency, warming up program, data processing. Thirdly, the control group is lacked. In this study, the control condition was the data 
collect at the initial time point, as we were looking at the time effect. To further enrich the study design, future studies can have a resting group added to further show the effect of participating and not participating in the prolonged football protocol.

In conclusion, the delayed peroneal muscle reaction was found after 30 minutes of the first half and 15 minutes of the second half of a football match. The risk of ankle sprain could increase in the latter minutes in each half protocol, so the prevention injury training strategies should focus on the specific duration in soccer match.

\section{Acknowledgement}

We acknowledged the participants of this study.

\section{Declaration of interest statement}

The authors declared no conflict of interest.

\section{Figure legends}

Figure 1: The position of EMG sensor during reaction time test

Figure 2: Reaction time of peroneal muscle during the prolonged football protocol $(*$ indicated significant difference from baseline $(0 \mathrm{~min}), \mathrm{p}<0.05)$.

\section{References}

1. Almonroeder, T.G., Tighe, S.M., Miller, T.M., \& Lanning, C.R. (2020) The influence of fatigue on decision-making in athletes: a systematic review. Sports Biomechanics, 19(1), 76-89.

2. Beynnon, B.D., Murphy, D.F., \& Alosa, D.M. (2002). Predictive factors for lateral ankle 
sprains: a literature review. Journal of Athletic Training, 37(4), 376-380.

3. Cohen, J. (2013). Statistical power analysis for the behavioral sciences. Routledge.

4. Delvaux, F., Schwartz, C., Rodriguez, C., Forthomme, B., Kaux, J.F., \& Croisier, J.L. (2020) Preseason assessment of anaerobic performance in elite soccer players: comparison of isokinetic and functional test. Sports Biomechanics. https://doi.org/10.1080/14763141.2020.1750681

5. de Noronha, M., Lay, E.K., McPhee, M.R., Mnatzaganian, G., \& Nunes, G.S. (2019). Ankle sprain has higher occurrence during the latter parts of matches: systematic review with meta-analysis. Journal of Sport Rehabilitation, 28(4), 373-380.

6. Doherty, C., Delahunt, E., Caulfield, B., Hertel, J., Ryan, J., Bleakley, C. (2014). The incidence and prevalence of ankle sprain injury: a systematic review and meta-analysis of prospective epidemiological studies. Sports Medicine, 44(1), 123-140.

7. Farquharson, C., \& Greig, M. (2017). Kinesiology tape mediates soccer- simulated and local peroneal fatigue in soccer players. Research in Sports Medicine, 25(3), 313-321.

8. Fong, D.T.P., Chan, Y.Y., Mok, K.M., Yung, P.S.H., \& Chan, K.M. (2009). Understanding acute ankle ligamentous sprain injury in sports. Sports Medicine, Arthroscopy, Rehabilitation, Therapy and Technology, 1, 14.

9. Fong, D.T.P., Hong, Y., Chan, L.K., Yung, P.S.H., \& Chan, K.M. (2007). A systematic review on ankle injury and ankle sprain in sports. Sports Medicine, 37(1), 73-94.

10. Fong, D.T.P., Leung, W.C., Mok, K.M., \& Yung, P.S.H. (2020). Delayed ankle muscle reaction time in female amateur footballers after the first 15 min of a simulated prolonged football protocol. Journal of Experimental Orthopaedics, 7, 54-62.

11. Fong, D.T.P., Wang, D., Chu, V.W.S., \& Chan, K.M. (2013). Myoelectric stimulation on peroneal muscles with electrodes of the muscle belly size attached to the upper shand gives the best effect in resisting simulated ankle sprain motion. Journal of Biomechanics, 
4(6), 1088-1091.

12. Gribble, P.A., Bleakley, C.M., Caulfield, B.M., Docherty, C.L., Fourchet, F., Fong, D.T.P., Hertel, J., Hiller, C.E., Kaminski, T.W., McKeon, P.O., Refshauge, K.M., Verhagen, E.A., Vicenzino, B.T., Wikstrom, E.A., \& Delahunt, E. (2016). Evidence review for the 2016 International Ankle Consortium consensus statement on the prevalence, impact and long-term consequences of lateral ankle sprains. British Journal of Sports Medicine, 50(24), 1496-1505.

13. Gribble, P.A., Hertel, J., \& Denegar, C.R. (2007). Chronic ankle instability and fatigue create proximal joint alterations during performance of the star excursion balance test. International Journal of Sports Medicine, 28(3), 236- 242.

14. Gullett, J. C., Tillman, M. D., Gutierrez, G. M., and Chow, J. W.(2009) .“A biomechanical comparison of back and front squats in healthy trained individuals," Journal of Strength and Conditioning Research, 23(1), 284-292.

15. Hakkinen, K. (1993). Neuromuscular fatigue and recovery in male and female athletes during heavy resistance exercise. International Journal of Sports Medicine, 14(2), 53-59.

16. Henry, B., McLoda, T., Docherty, C.L., \& Schrader, J. (2010). The effect of plyometric training on peroneal latency. Journal of Sport Rehabilitation, 19(3), 288-300.

17. Hertel, J. (2002). Functional anatomy, pathomechanics, and pathophysiology of lateral ankle instability. Journal of Athletic Training, 37(4), 364-375.

18. Hoch, M.C., \& McKeon, P.O. (2014). Peroneal reaction time after ankle sprain: a systematic review and meta-analysis. Medicine and Science in Sports and Exercise, 46(3), 546-56.

19. Jackson, N.D., Gutierrez, G.M., \& Kaminski, T. (2009). The effect of fatigue and habituation on the stretch reflex of the ankle musculature. Journal of Electromyography and Kinesiology, 19(1), 75-84. 
20. Knight, A.C., \& Weimar, W.H. (2011). Difference in response latency of the peroneus longus between the dominant and nondominant legs. Journal of Sport Rehabilitation, 20(3), 321-32.

21. Konrad, P. (2006). The ABC of EMG: a practical introduction to kinesiological electromyography. Noraxon Inc., USA, Version 1.0.

22. Konradsen, L., \& Ravn, J.B. (1991). Prolonged peroneal reaction time in ankle instability. International Journal of Sports Medicine, 12(3), 290-292.

23. Lamb, S.E., March, J.L., Hutton, J.L., Nakash, R., Cooke, M.W., Collaborative Ankle Support Trial (CAST Group) (2009). Mechanical supports for acute, severe ankle sprain: a pragmatic, multicentre, randomised controlled trial. Lancet, 373(9663), 575-581.

24. Merletti, R. \& Torino P. D.(1999). Standards for reporting EMG data, Journal of Electromyography and Kinesiology,9(1):3-4.

25. Nicholas, C.W., Nuttall, F.E., \& Williams, C. (2000). The Loughborough intermittent shuttle test: a field test that simulates the activity pattern of soccer. Journal of Sports Sciences, 18(2), 97-104.

26. Pavin, L.N., Leicht, A.S., Gimenes, S.V., da Silva, V.C., de Moura Simim, M.A., Marocolo, M., \& da Moto, G.R. (2019). Can compression stockings reduce the degree of soccer match-induced fatigue in females? Research in Sports Medicine, 27(3), 351-364.

27. Perotto, A.O. (2005). Anatomical guide for the electromyographer: the limb and trunk (4th ed.). Springfield, IL 62704: Charles C Thomas Publisher Ltd, 175-192.

28. Pierce, C.A., Block, R.A., \& Aguinis, H. (2004). Cautionary note on reporting etasquared values from multifactor ANOVA designs. Educational and Psychological Measurement, 64(6), 916-924.

29. Rahnama, N., Lees, A., \& Reilly, T. (2006). Electromyography of selected lower-limb muscles fatigued by exercise at the intensity of soccer match-play. Journal of 
Electromyography and Kinesiology, 16(3), 257-263.

30. Reilly, T., Drust, B., \& Clarke, N. (2008). Muscle fatigue during football match-play. Sports Medicine, 38(5), 357-367.

31. Sun, W., Song, Q., Yu, B., Zhang, C., \& Mao, D. (2015). Test-retest reliability of a new device for assessing ankle joint threshold to detect passive movement in healthy adults. Journal of Sports Sciences, 33(16), 1667-1674.

32. Sun, W., Zhang, C, Song, Q, Li, W., Cong, Y., Chang, W., Mao, D., \& Hong, Y. (2016). Effect of 1-year regular Tai Chi on neuromuscular reaction in elderly women: a randomized controlled study. Research in Sports Medicine, 24(2), 145-56.

33. Valldecabres, R., Richards, J., \& De Benito, A.M. (2020) The effect of match fatigue in elite badminton players using plantar pressure measurements and the implications to injury mechanisms. Sports Biomechanics. https://doi.org/10.1080/14763141.2020.1712469

34. Xu, D.Q., Li, J.X., Hong, Y. (2005). Effect of regular Tai Chi and jogging exercise on neuromuscular reaction in older people. Age and Ageing, 34(5), 439-444.

35. Yeung, M.S., Chan, K.M., So, C.H., \& Yuan, W.Y. (1994). An epidemiological survey on ankle sprain. British Journal of Sports Medicine, 28(2), 112-116. 


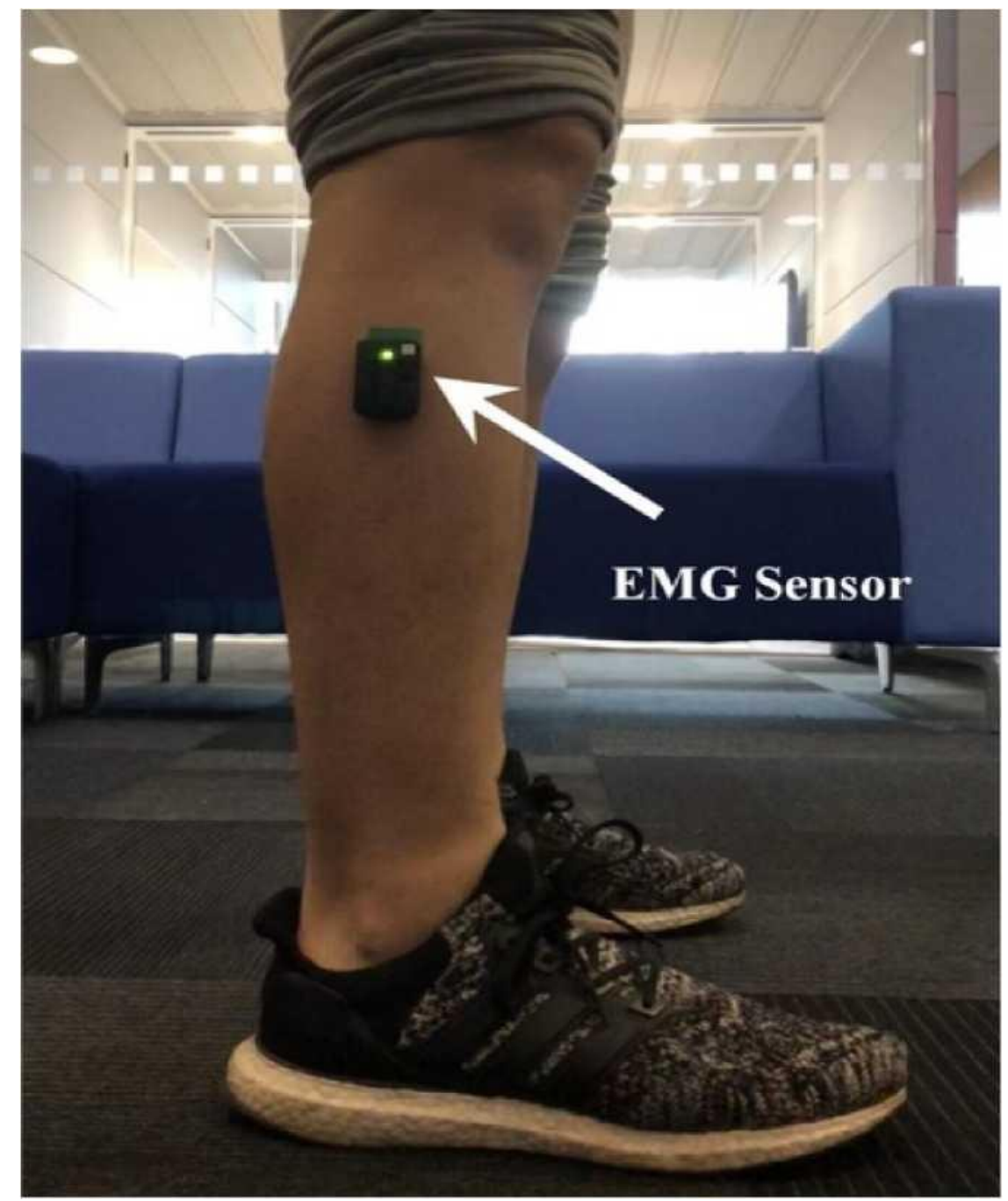

Figure 1: The position of EMG sensor during reaction time test 
Reaction time (ms) of peroneal muscle during the prolonged football protocol

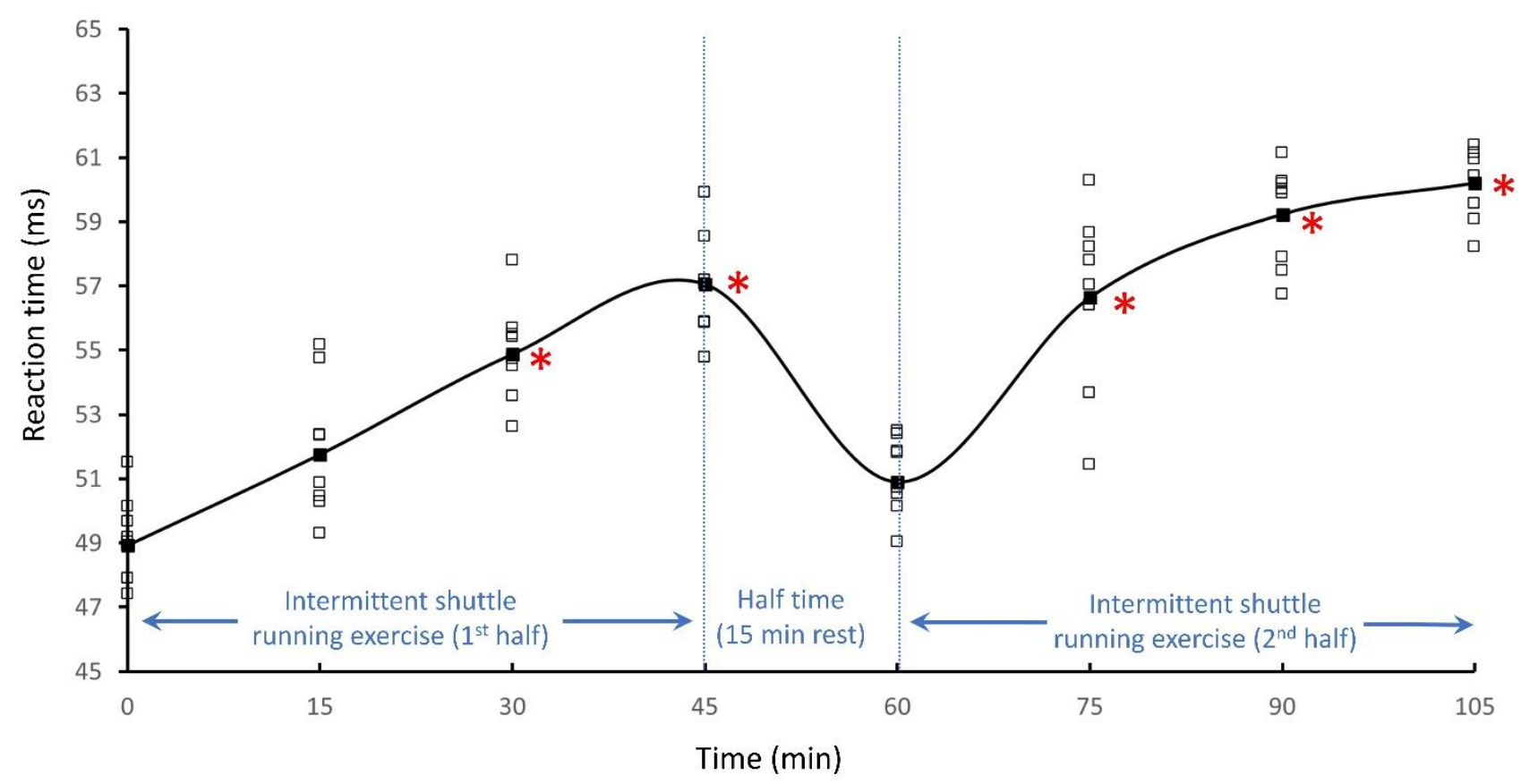

Figure 2: Reaction time of peroneal muscle during the prolonged football protocol $(*$ indicated significant difference from baseline ( $0 \mathrm{~min}), \mathrm{p}<0.05)$. 
Table 1: The reaction time of peroneal muscle throughout the prolonged simulated football protocol.

\begin{tabular}{lllll}
\hline Time $(\min )$ & Reaction Time $(\mathrm{ms})$ & $\mathrm{p}$-value of t-test with baseline & $95 \%$ CI for difference & Cohen's d \\
\hline 0 min & $48.9 \pm 1.3$ & - & - & - \\
$15 \mathrm{mins}$ & $51.8 \pm 2.2$ & 1.000 & $-8.219,2.564$ & 1.57 \\
$30 \mathrm{mins}$ & $54.9 \pm 1.6$ & $0.001^{*}$ & $-8.853,-3.044$ & 4.05 \\
$45 \mathrm{mins}$ & $57.1 \pm 1.7$ & $<0.001^{*}$ & $-9.627,-6.606$ & 5.37 \\
$60 \mathrm{mins}$ & $50.9 \pm 1.3$ & 0.522 & $-5.127,1.187$ & 1.53 \\
$75 \mathrm{mins}$ & $56.6 \pm 3.0$ & $0.005^{*}$ & $-13.024,-2.401$ & 3.37 \\
$90 \mathrm{mins}$ & $59.2 \pm 1.6$ & $<0.001^{*}$ & $-14.430,-6.178$ & 6.91 \\
$105 \mathrm{mins}$ & $60.2 \pm 1.1$ & $<0.001^{*}$ & $-14.152,-8.391$ & 9.13 \\
\hline
\end{tabular}
$*$, significant difference from baseline $(0 \mathrm{~min}), \mathrm{p}<0.05$. 